\title{
Identification of potential therapeutic targets for melanoma using gene expression analysis
}

\author{
L. QUAN, J. SHI, Y. TIAN, Q. ZHANG, Y. ZHANG, Y. ZHANG, Q. HUI, K. TAO*
}

Department of Plastic Surgery, General Hospital of Shenyang Military Area Command, PLA83. Wenhua Road, Shenhe District, Shenyang, Liaoning, China 110016

*Correspondence: kaitaoktkt@163.com

Received January 15, 2015 / Accepted March 30, 2015

\begin{abstract}
Metastatic melanoma represents a significant cause of death in patients with melanoma and the frequency is increasing. The aim of this study was to identify potential therapeutic targets for metastatic melanoma.

Gene expression profile GSE44660 was downloaded from Gene Expression Omnibus database. A total of 22 samples were analyzed in our study, including 3 specimens of normal melanocytes, 12 specimens of melanoma LNM (lymph node metastasis) and 7 specimens of MBM (melanoma brain metastasis). DEGs (differentially expressed genes) in LNM and MBM were identified respectively using Limma package. GO (Gene Ontology) and KEGG (Kyoto Encyclopedia of Genes and Genomes) pathways analyses of common DEGs between two comparison groups were performed using DAVID, followed by cancer-related genes and transcription factor analysis. PPI (protein-protein interaction) network was constructed by STRING, and significant key genes were selected.

Totally, 401 common DEGs were identified. Disease analysis showed that ICAM1 (intercellular adhesion molecule 1) and NBN (nibrin) were related to melanoma. In the PPI network, BIRC5 (baculoviral IAP repeat containing 5), BUB1 (BUB1 mitotic checkpoint serine/threonine kinase), GMNN (geminin, DNA replication inhibitor), AURKA (aurora kinase A), TOP2A (topoisomerase (DNA) II alpha) and BUB1B (BUB1 mitotic checkpoint serine/threonine kinase B) were with higher degree more than 50 .

ICAM1, NBN, BIRC5, BUB1, BUB1B, GMNN, AURKA and TOP2A may play key roles in the progression and development of melanoma. They may be used as specific therapeutic targets in the treatment of metastatic melanoma. However, further experiments are still needed to confirm our results.
\end{abstract}

Key words: melanoma, metastasis, differentially expressed gene, protein-protein interaction network

Melanoma is considered as a type of skin cancer that originated from melanocytes [1]. High incidence is particularly in Caucasians, especially northwestern and northern Europeans, who live in sunny climates [2]. In 2012, the estimated rates of new melanoma cases in Europe are 11.0 per 100,000 for females and 11.4 per 100,000 men [3]. Metastatic

\footnotetext{
Abbreviations: BP - biological process; CC - cell component; DAVID - Database for Annotation, Visualization and Integrated Discovery; DEGs - differentially expressed genes; FC - fold change; FDR - false discovery rate; GO - Gene Ontology; ICAM1 - intercellular adhesion molecule 1; KEGG - Kyoto Encyclopedia of Genes and Genomes; LNM - lymph node metastasis; MAPK/ERK - mitogen-activated protein kinase/extracellular regulated kinase; MBM - melanoma brain metastasis; MF - molecular function; PPI - protein-protein interaction; STRING - Search Tool for the Retrieval of Interacting Genes
}

melanoma represents a significant cause of death in patients with melanoma and the frequency is increasing. Primary melanoma can commonly metastasize to regional and distant lymph nodes and then by hematogenic dissemination into distant organs including liver, brain and lung [4]. Despite the advances in surgery, it causes the majority of deaths (75\%) related to skin cancer [5]. Therefore, it is essential to develop more effective methods for its treatment.

Recently, an increasing number of studies have focused on the prevention and treatment of melanoma, and then found that targeted therapy trials are promising. Carvajal et al. have shown that KIT (v-kit Hardy-Zuckerman 4 feline sarcoma viral oncogene homolog) is a therapeutic target in metastatic melanoma [6]. Notably, the Ras/Raf/MEK/ ERK pathway has been considered as a major, druggable 
regulator of melanoma [7]. BRAF (v-raf murine sarcoma viral oncogene homolog B) mutation is commonly observed in human melanoma, which resulting in melanocyte hyperproliferation and overactive of MAPK/ERK (Mitogenactivated protein kinase/Extracellular Regulated Kinase) signaling [8]. Nevertheless, resistance to BRAF inhibitors is a major clinical challenge. Moreover, Ericsson et al. have found that IGF1R (insulin-like growth factor-1 receptor) is a predictor for metastatic disease and a potential therapeutic target [9]. Cotargeting MEK and IGF1R/PI3K can overcome resistance to BRAF inhibitors [10]. Rappa et al. have indicated that the stem cell-associated antigen CD133 is a molecular therapeutic target for metastatic melanoma [11]. Although tremendous efforts have been made to discover novel targets for melanoma treatment and novel targeted therapies are showing promising, resistance to these agents and patient relapse rapidly ensue. Therefore, more novel therapeutic targets for metastatic melanoma into brain and lymph node are needed to improve clinical treatment.

DNA methylation profiling associated with MBM (melanoma brain metastasis) and LNM (lymph node metastasis) have been performed by Marzese and colleagues [12]. In this study, DEGs (differentially expressed genes) in MBM and LNM compared with normal controls were identified respectively. Then function annotation of common genes between two comparison groups was conducted, followed by PPI (protein-protein interaction) network construction and key genes screening. Through the identification of key genes, the possible molecular mechanism and the potential therapeutic targets for melanoma were explored. The aim of this study was to identify novel gene targets to improve metastatic melanoma therapy.

\section{Materials and methods}

Affymetrix microarray data. We obtained the gene expression profile data GSE44660 from Gene Expression Omnibus database (http://www.ncbi.nlm.nih.gov/geo/) which was deposited by Marzese et al. [12]. The gene expression profiling was based on the platform of GPL5175 ([HuEx-1_0-st] Affymetrix Human Exon 1.0 ST Array). Totally, 22 samples were analyzed in our study, including 3 specimens of normal melanocytes, 12 specimens of melanoma LNM and 7 specimens of MBM.

Identification of the common DEGs. The raw data were preprocessed using the Affy package (http://www. bioconductor.org/packages/release/bioc/html/affy.html) [13] in R language. DEGs in melanoma LNM and DEGs in MBM compared with normal melanocytes were screened respectively by Limma package (http://www.bioconductor. org/packages/release/bioc/html/limma.html) in R [14]. The multiple testing correction was performed to control the FDR (false discovery rate) [15]. FC (fold change) of individual gene was also calculated. The genes with FDR $<0.01$ and $\log _{2}|\mathrm{FC}|$ $\geq 1$ were considered to be significant. Finally, the common DEGs between two comparison groups were obtained.
Functional and pathway enrichment analysis of DEGs. The GO (Gene ontology, http://geneontology.org/) functional analysis has become a commonly used approach for functional studies of large-scale genomic or transcriptomic data [16]. KEGG (Kyoto Encyclopedia of Genes and Genomes, http:// www.genome.jp/kegg/pathway.html) pathway database [17] contains information of graphical diagrams of biological pathways including some of the known regulatory pathways and most of known metabolic pathways. DAVID (Database for annotation visualization and integrated discovery, http:// david.abcc.ncifcrf.gov/) online tool [18] was applied to systematically extract biological meanings from large gene or protein lists. GO function in BP (biological process), CC (cell component), MF (molecular function) and KEGG pathway enrichment analysis of common DEGs were performed using DAVID (version 6.7) with $P<0.05$.

Analysis of cancer-related genes. Genetic-association-DBDisease analysis was performed by uploading the common DEGs to DAVID system. Then, cancer-related genes were screened out, and heatmap of cancer-related genes was generated using the heatmap.2 function of the "gplots" package in R (http://www.inside-r.org/packages/cran/gplots/docs/ heatmap.2) [19].

PPI network construction. The STRING (search tool for the retrieval of interacting genes, http://string-db.org/) [20] database was used to retrieve the predicted interactions for the common DEGs. All interactions obtained from STRING are provided with a confidence score, and each score represents a rough estimate of how likely a given association describes a functional linkage between two proteins [21]. The common DEGs with a confidence score more than 0.4 including cancer-related genes were selected to construct the PPI network, visualized using the Cytoscape software (http:// cytoscape.org/) [22].

Transcription factor analysis. The online tool Whole Genome RVista [23] \{Zambon, 2005 \#1006\}(http://genome. lbl.gov/cgi-bin/WGRVistaInput5.pl?cfg_dir=gp_r4099_169), a publicly available bioinformatics program, was used to analyze the significantly enriched transcription factor binding sites in the regulatory promoter region of cancer related genes (5000 bp upstream of the transcriptional start sites). The transcription factor with $P \leq 0.1$ was screened out. Then, the network including transcription factor and cancer-related genes was visualized using Cytoscape software

\section{Results}

Functional and pathway enrichment analysis of common DEGs. A total of 401 common DEGs between two comparison groups including 352 up-regulated genes and 49 down-regulated genes were identified (Figure 1). Functional enrichment analysis showed that the up-regulated DEGs were significantly enriched in GO terms of RNA processing, nuclear division, mitosis and $\mathrm{M}$ phase of mitotic cell cycle (Table 1); the down-regulated DEGs were mainly involved in regulation 
of cell development, negative regulation of cell differentiation and embryonic appendage morphogenesis (Table 2). Pathway enrichment analysis showed that the up-regulated DEGs were significantly enriched in spliceosome and pyrimidine metabolism (Table 1). However, no significant pathway of down-regulated DEGs was identified.

Analysis of cancer-related genes. The functions of these common DEGs were preliminarily investigated by DAVID online servers under the Genetic Association DB Disease. The results revealed that 18 out of 401 common genes were significantly associated with cancers (Figure 2). Moreover, most of DEGs were up-regulated in both two comparison groups excluding down-regulated EPHX1 (epoxide hydrolase 1, microsomal). Especially, only two genes of ICAM1 (intercellular adhesion molecule 1) and NBN (nibrin) were related to melanoma.

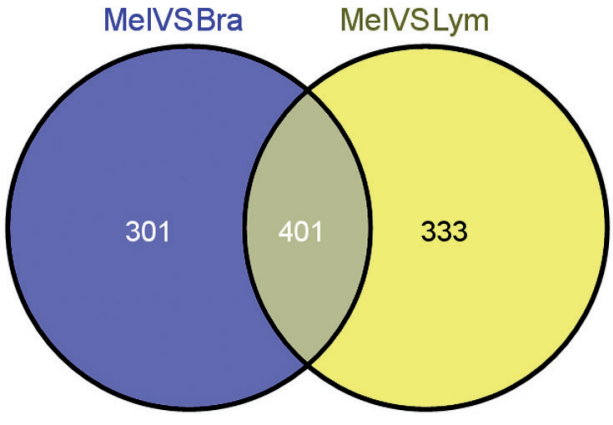

Figure 1. The venn diagram of selected differentially expressed genes (DEGs) in melanoma brain metastasis (MBM) and lymph node metastasis (LNM). MelVSBra indicates DEGs between normal melanocytes and MBM; MelVSLym indicates DEGs between normal melanocytes and melanoma LNM. The overlapped genes were common DEGs between two comparison groups.

Table 1. Gene ontology (GO) functional and pathway enrichment analysis of the up-regulated differentially expressed genes.

\begin{tabular}{|c|c|c|c|}
\hline Category & Term & Count & $P$ Value \\
\hline GOTERM_BP_FAT & GO:0006396 RNA processing & 49 & $4.94 \mathrm{E}-19$ \\
\hline GOTERM_BP_FAT & GO:0000280 nuclear division & 30 & $2.14 \mathrm{E}-16$ \\
\hline GOTERM_BP_FAT & GO:0007067 mitosis & 30 & $2.14 \mathrm{E}-16$ \\
\hline GOTERM_BP_FAT & GO:0000087 M phase of mitotic cell cycle & 30 & $3.48 \mathrm{E}-16$ \\
\hline GOTERM_BP_FAT & GO:0048285 organelle fission & 30 & $7.42 \mathrm{E}-16$ \\
\hline GOTERM_CC_FAT & GO:0043232 intracellular non-membrane-bounded organelle & 127 & $6.86 \mathrm{E}-28$ \\
\hline GOTERM_CC_FAT & GO:0043228 non-membrane-bounded organelle & 127 & $6.86 \mathrm{E}-28$ \\
\hline GOTERM_CC_FAT & GO:0031981 nuclear lumen & 93 & $2.96 \mathrm{E}-27$ \\
\hline GOTERM_CC_FAT & GO:0070013 intracellular organelle lumen & 103 & $4.40 \mathrm{E}-27$ \\
\hline GOTERM_CC_FAT & GO:0043233 organelle lumen & 103 & $2.81 \mathrm{E}-26$ \\
\hline GOTERM_MF_FAT & GO:0003723 RNA binding & 42 & $7.20 \mathrm{E}-11$ \\
\hline GOTERM_MF_FAT & GO:0008135 translation factor activity, nucleic acid binding & 11 & $1.26 \mathrm{E}-05$ \\
\hline GOTERM_MF_FAT & GO:0003743 translation initiation factor activity & 9 & $1.49 \mathrm{E}-05$ \\
\hline GOTERM_MF_FAT & GO:0000166 nucleotide binding & 66 & $7.70 \mathrm{E}-05$ \\
\hline GOTERM_MF_FAT & GO:0032555 purine ribonucleotide binding & 53 & $8.38 \mathrm{E}-04$ \\
\hline KEGG_PATHWAY & hsa03040:Spliceosome & 12 & 4.39E-06 \\
\hline KEGG_PATHWAY & hsa00240:Pyrimidine metabolism & 7 & 0.004055211 \\
\hline
\end{tabular}

BP: biological process; CC: biological process; MF: molecular function; Count: numbers of DEGs in each GO term; KEGG: Kyoto Encyclopedia of Genes and Genomes.

Table 2. Gene ontology (GO) functional enrichment analysis of the down-regulated differentially expressed genes.

\begin{tabular}{llcl}
\hline Category & Term & P Value & Count \\
\hline GOTERM_BP_FAT & GO:0060284 regulation of cell development & 4 \\
GOTERM_BP_FAT & GO:0045596 negative regulation of cell differentiation & 4 \\
GOTERM_BP_FAT & GO:0035113 embryonic appendage morphogenesis & 0.01339419 & 3 \\
GOTERM_BP_FAT & GO:0030326 embryonic limb morphogenesis & 3 \\
GOTERM_BP_FAT & GO:0035108 limb morphogenesis & 3 \\
GOTERM_CC_FAT & GO:0016023 cytoplasmic membrane-bounded vesicle & 0.018971394 & 0.024158796 \\
GOTERM_CC_FAT & GO:0031988 membrane-bounded vesicle & 0.007299266 \\
GOTERM_CC_FAT & GO:0031410 cytoplasmic vesicle & 0.008338855 \\
GOTERM_CC_FAT & GO:0031982 vesicle & 0.013712789 & 6 \\
GOTERM_CC_FAT & GO:0016021 integral to membrane & 6 \\
GOTERM_MF_FAT & GO:0008134 transcription factor binding & 0.016251043 \\
\hline
\end{tabular}

BP: biological process; CC: biological process; MF: molecular function; Count: numbers of DEGs in each GO term. 


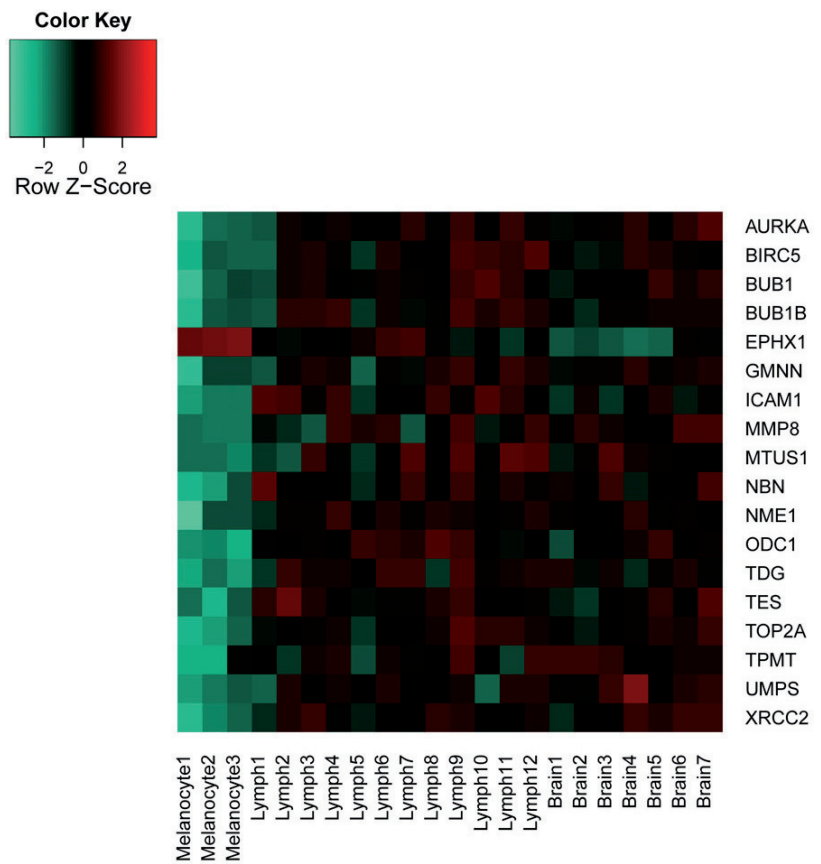

Figure 2. Heatmap of cancer-related genes.

Average gene expression value was $\log 2$ converted. Each row indicates the common differentially expressed gene (DEG); each column indicates the sample. $>2$ Red for high expression in metastatic melanoma compared with normal control and $<2$ green for low expression in metastatic melanoma compared with normal control.
PPI network and transcription factor analysis. In the PPI network (Figure 3), most of genes interacted with cancerassociated genes were up-regulated. Six cancer-associated genes including BUB1 (BUB1 mitotic checkpoint serine/threonine kinase), GMNN (geminin, DNA replication inhibitor), BIRC5 (baculoviral IAP repeat containing 5), AURKA (aurora kinase A), TOP $2 A$ (topoisomerase (DNA) II alpha) and $B U B 1 B$ (BUB1 mitotic checkpoint serine/threonine kinase B) with higher degree more than 50 were identified as key genes in metastatic melanoma. Transcription factor analysis showed the lrx2 (iroquois homeobox 2) was the key transcription factor regulating cancer-related genes $A U R K A$ and $B U B 1 B$ (Figure 4).

\section{Discussion}

Melanoma causes the majority of deaths related to skin cancer [5]. Now, the large health burden of melanoma in population is enormous [24]. Thus, the potential use of new therapeutic targets appears to be the most promising area of research. In this work, we used bioinformatics approaches to predict the potential therapeutic targets for melanoma, and 401 common DEGs between two comparison groups were screened out. In addition to the known melanoma-related genes ICAM1 and $N B N$, six abnormally expressed genes including BIRC5, BUB1, BUB1B, GMNN, AURKA and TOP2A were identified to be key genes that may play an important role in melanoma metastasis.

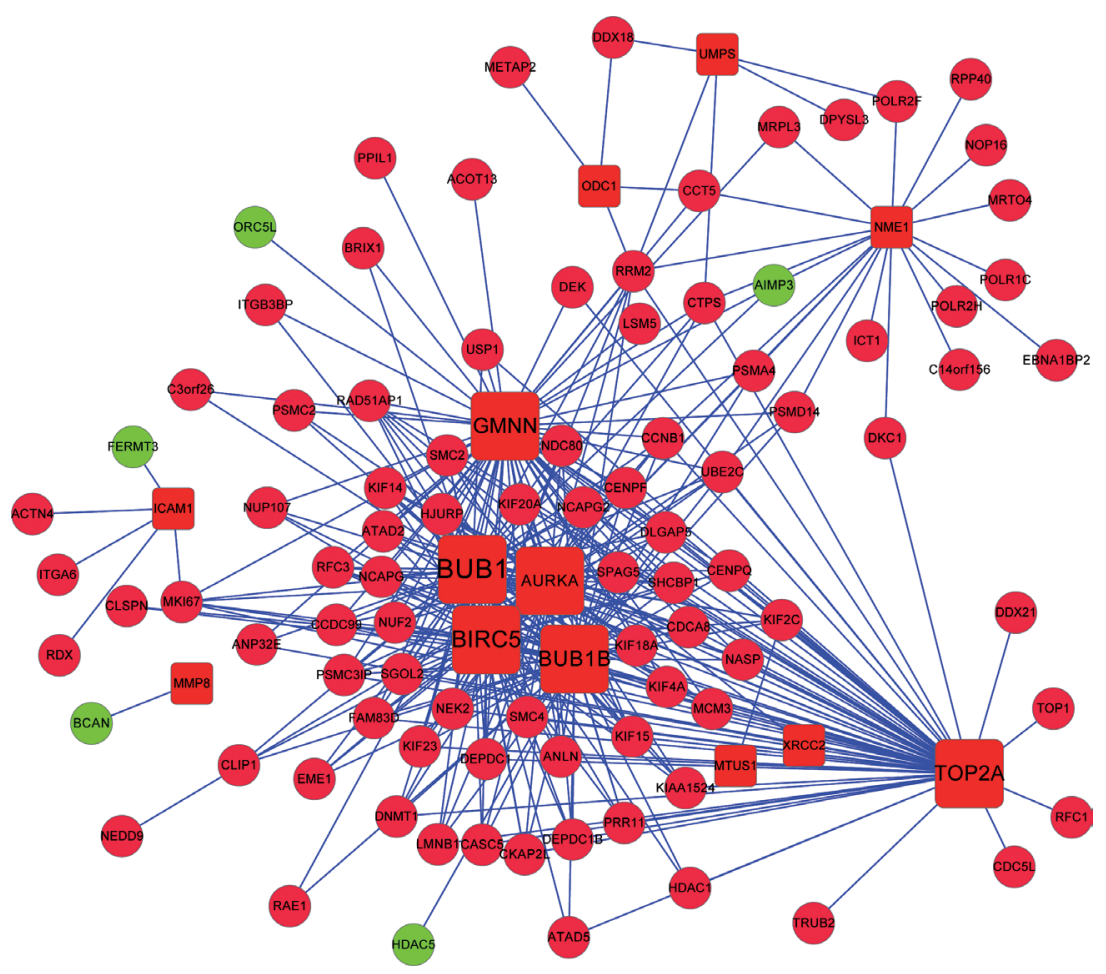

Figure 3. The protein-protein interaction network of common genes in metastatic menaloma.

Red nodes represent up-regulated genes and green nodes represent down-regulated genes. Square nodes represent cancer-related genes and large square nodes represent key genes in metastatic melanoma. 
ICAM1 encodes a cell surface glycoprotein which is typically expressed in endothelial cells [25]. Johnson et al. have found that the increased expression of ICAM-1 on melanoma cells may positively correlated with a greater risk of metastasis [26]. Moreover, Banks and colleagues have found that levels of ICAM1 were significantly increased in melanoma, and $I C A M-1$ has been implicated in tumor progression and metastasis [27]. Kageshita et al. have suggested that monitoring of serum ICAM-1 level may contribute to monitor the clinical course of the malignant melanoma [28]. Additionally, another known melanoma-related gene $N B N$ has been proved to be overexpressed in aggressive uveal melanoma, and thus considered as a prognostic marker [29]. In our studies, ICAM-1 and NBN were up-regulated and related to metastatic melanoma in Genetic-association-DB-Disease analysis which was consistent with the previous study, therefore, ICAM-1 and $N B N$ may be a therapeutic target in metastatic melanoma.

In PPI network, six genes with higher degree more than 50 , including BUB1, GMNN, BIRC5, AURKA, TOP $2 A$ and $B U B 1 B$ were identified to be key novel genes in metastatic melanoma. $B U B 1$ and $B U B 1 B$ encode a serine/threonine-protein kinase that plays a central role in mitosis [30]. In a previous study, Grabsch et al. have found that overexpression of BUB1 and $B U B 1 B$ in gastric cancer may contribute to tumor cell proliferation [30]. Pinto et al. have shown that overexpression of $B U B 1$ is associated with genomic complexity in clear cell kidney carcinomas [30]. Ricke et al. have proved that $B U B 1$ overexpression induces tumor formation through Aurora B kinase hyperactivation [31]. Notably, higher levels of $B U B 1$ has been shown in metastatic melanomas than primary melanoma indicating the key role of BUB1 in metastasis of melanoma [32]. Disappointed, few studies have reported the association between $B U B I B$ and metastatic melanoma. In our study, $B U B 1$ and $B U B 1 B$ were up-regulated, and mainly involved in mitosis biological process which was consistent with previous study. As a result, $B U B 1$ and $B U B 1 B$ might play vital role in melanoma metastasis and be predicted therapeutic targets.

BIRC5 is a member of inhibitor of apoptosis gene family and product preventing apoptotic cell death [33]. Abnormal expression of BIRC5 has been proved to be associated with prognosis and metastasis of melanoma [34]. Importantly, survivin, an anti-apoptotic protein encoded by BIRC5, has been shown to be correlated with progression in human melanoma and LNM [35]. GMNN encodes a protein that plays a critical role in cell cycle regulation [36]. Increased expression of GMNN may play a role in several malignancies including colon, rectal and breast cancer [37]. More recently, GMNN has been observed to be overexpressed in distant metastatic patients and is correlated with prognosis of melanoma [34]. AURKA overexpression may play a role in tumor development and progression via promoting cancer cell survival, stimulating the PI3K pathway and activating Akt [38]. Additionally, AU$R K A$ has been down-regulated in differentiation of metastatic HO-1 human melanoma cells, and therefore contributes to

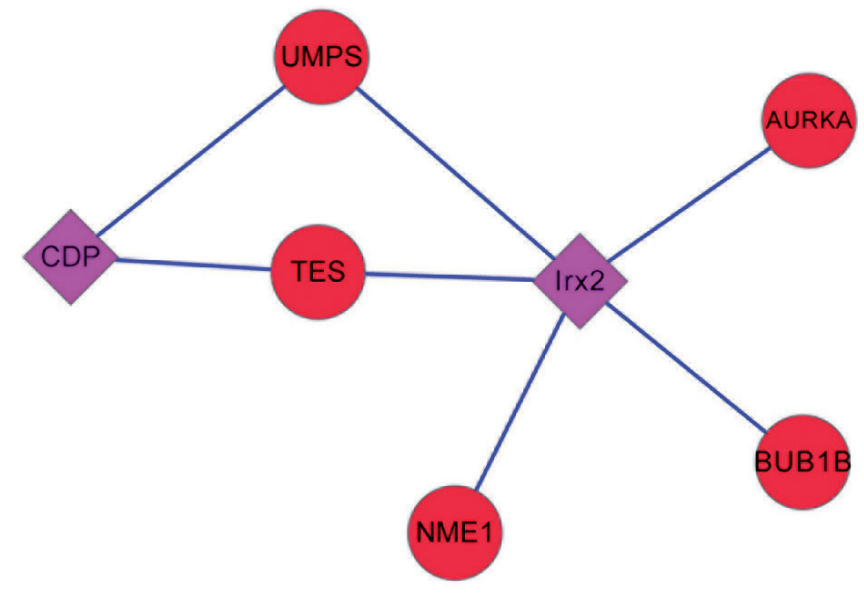

Figure 4. The transcription factor regulatory network.

The diamond nodes represent the transcription factors and the red circle nodes represent up-regulated genes.

HO-1 cell proliferation [39]. Notably, TOP2A is considered as an oncogene in tumor and significantly involved in cell cycle, thus associated with tumor cell growth, proliferation and migration [40]. Particularly, TOP $2 A$ has been shown to be overexpressed in patients with metastatic melanoma [41]. In our studies, up-regulated BIRC5, GMNN, AURKA and TOP2A may be obviously involved in the melanoma development through regulating cell growth, and thus, GMNN, AURKA and TOP $2 A$ may be considered to be therapeutic targets in melanoma. Furthermore, transcription factor Irx2 regulated expression of AURKA and BUB1B. As we all know, vertebrate Irx genes are involved in neural development [42]. Specifically, Irx2 is shown to be involved in cerebellum formation through regulating FGF8/MAP kinase signaling pathway [43]. As a result, transcription factor Irx2 may play a key role in MBM by targeting AURKA and BUB1B.

There are some limitations in this study. First, the sample size for microarray analysis is relatively small. Other datasets will be collected in further study. Second, the genes identified in our preliminary study are required to be further confirmed by RT-PCR or western blot. As a result, further experimental studies based on a larger sample size are needed to validate our results.

\section{Conclusions}

In conclusion, the ICAM1, NBN, BIRC5, BUB1, BUB1B, GMNN, AURKA and TOP2A may play key roles in the progression and development of melanoma. They might be specific therapeutic targets in the treatment of melanoma. This report represents a comprehensive gene expression analysis of melanoma metastasis and opens up new avenues to identify novel molecular markers and therapeutic targets for metastatic melanoma therapy. However, further experiments are still needed to confirm our results. 


\section{References}

[1] FLAHERTY KT, HODI FS, BASTIAN BC. Mutation-driven drug development in melanoma. Curr Opin Oncol 2010; 22: 178. http://dx.doi.org/10.1097/CCO.0b013e32833888ee

[2] PARKIN DM, BRAY F, FERLAY J, PISANI P. Global cancer statistics, 2002. CA Cancer J Clin 2005; 55: 74-108. http:// dx.doi.org/10.3322/canjclin.55.2.74

[3] NIKOLAOU V, STRATIGOS A. Emerging trends in the epidemiology of melanoma. Brit J of Dermatol 2014; 170: 11-19. http://dx.doi.org/10.1111/bjd.12492

[4.] MILLER AJ, MIHM JR MC. Melanoma. New Engl J Med 2006; 355: 51-65. http://dx.doi.org/10.1056/NEJMra052166

[5] JERANT AF, JOHNSON JT, SHERIDAN CD, CAFFREY TJ. Early detection and treatment of skin cancer. Am Fam Physician 2000; 62: 357-368, 375-356, 381-352.

[6] CARVAJAL RD, ANTONESCU CR, WOLCHOK JD, CHAPMAN PB, ROMAN R-A et al. KIT as a therapeutic target in metastatic melanoma. JAMA 2011; 305: 2327-2334. http:// dx.doi.org/10.1001/jama.2011.746

[7] COHEN C, ZAVALA-POMPA A, SEQUEIRA JH, SHOJI M, SEXTON DG et al. Mitogen-actived protein kinase activation is an early event in melanoma progression. Clin Cancer Res 2002; 8: 3728-3733.

[8] GRAY-SCHOPFER VC, DA ROCHA DIAS S, MARAIS R. The role of B-RAF in melanoma. Cancer Metast Rev 2005; 24: 165-183. http://dx.doi.org/10.1007/s10555-005-5865-1

[9] ALL-ERICSSON C, GIRNITA L, SEREGARD S, BARTOLAZZI A, JAGER MJ et al. Insulin-like growth factor-1 receptor in uveal melanoma: a predictor for metastatic disease and a potential therapeutic target. Invest Ophth Vis Sci 2002; 43: $1-8$.

[10] VILLANUEVA J, VULTUR A, LEE JT, SOMASUNDARAM R, FUKUNAGA-KALABIS $M$ et al. Acquired resistance to BRAF inhibitors mediated by a RAF kinase switch in melanoma can be overcome by cotargeting MEK and IGF-1R/PI3K. Cancer Cell 2010; 18: 683-695. http://dx.doi.org/10.1016/j. ccr.2010.11.023

[11] RAPPA G, FODSTAD O, LORICO A. The Stem Cell-Associated Antigen CD133 (Prominin-1) Is a Molecular Therapeutic Target for Metastatic Melanoma. Stem Cells 2008; 26: 30083017. http://dx.doi.org/10.1634/stemcells.2008-0601

[12.] MARZESE DM, SCOLYER RA, HUYNH JL, HUANG SK, HIROSE $\mathrm{H}$ et al. Epigenome-wide DNA methylation landscape of melanoma progression to brain metastasis reveals aberrations on homeobox D cluster associated with prognosis. Hum Mol Genet 2013: ddt420.

[13] GAUTIER L, COPE L, BOLSTAD BM, IRIZARRY RA. affyanalysis of Affymetrix GeneChip data at the probe level. Bioinformatics 2004; 20: 307-315. http://dx.doi.org/10.1093/ bioinformatics/btg 405

[14] SMYTH GK. Limma: linear models for microarray data. In: Huber RGAVCASDARIAW, editor. Bioinformatics and Computational Biology Solutions Using $\{\mathrm{R}\}$ and Bioconductor. New York: Springer; 2005. p. 397--420.

[15] YEKUTIELI D, BENJAMINI Y. Resampling-based false discovery rate controlling multiple test procedures for correlated test statistics. J Stat Plan Infer 1999; 82: 171-196. http://dx.doi. org/10.1016/S0378-3758(99)00041-5

[16] HULSEGGE I, KOMMADATH A, SMITS MA. Globaltest and GOEAST: two different approaches for Gene Ontology analysis. BMC Proc 2009; 3 Suppl 4: S10. http://dx.doi. org/10.1186/1753-6561-3-s4-s10

[17] OGATA H, GOTO S, SATO K, FUJIBUCHI W, BONO H et al. KEGG: Kyoto Encyclopedia of Genes and Genomes. Nucleic Acids Res 1999; 27: 29-34. http://dx.doi.org/10.1093/ nar/27.1.29

[18] DENNIS JR G, SHERMAN BT, HOSACK DA, YANG J, GAO W et al. DAVID: database for annotation, visualization, and integrated discovery. Genome Biol 2003; 4: P3. http://dx.doi. org/10.1186/gb-2003-4-5-p3

[19] LIGGES U, M CHLER M. Scatterplot3d-an r package for visualizing multivariate data: Technical Report, SFB 475: Komplexitätsreduktion in Multivariaten Datenstrukturen, Universität Dortmund2002.

[20] FRANCESCHINI A, SZKLARCZYK D, FRANKILD S, KUHN M, SIMONOVIC $M$ et al. STRING v9.1: proteinprotein interaction networks, with increased coverage and integration. Nucleic Acids Res 2013; 41: D808-815. http:// dx.doi.org/10.1093/nar/gks1094

[21] SZKLARCZYK D, FRANCESCHINI A, KUHN M, SIMONOVIC M, ROTH A et al. The STRING database in 2011: functional interaction networks of proteins, globally integrated and scored. Nucleic Acids Res 2011; 39: D561D568. http://dx.doi.org/10.1093/nar/gkq973

[22] SHANNON P, MARKIEL A, OZIER O, BALIGA NS, WANG JT et al. Cytoscape: a software environment for integrated models of biomolecular interaction networks. Genome Res 2003; 13: 2498-2504. http://dx.doi.org/10.1101/gr.1239303

[23] ZAMBON AC, ZHANG L, MINOVITSKY S, KANTER JR, PRABHAKAR $S$ et al. Gene expression patterns define key transcriptional events in cell-cycle regulation by cAMP and protein kinase A. P Natl Acad Sci USA 2005; 102: 8561-8566. http://dx.doi.org/10.1073/pnas.0503363102

[24.] HARRIS NL, JAFFE ES, DIEBOLD J, FLANDRIN G, MULLER-HERMELINK HK et al. World Health Organization classification of neoplastic diseases of the hematopoietic and lymphoid tissues: report of the Clinical Advisory Committee meeting-Airlie House, Virginia, November 1997. J Clin Oncol 1999; 17: 3835-3849.

[25] RICE GE, BEVILACQUA MP. An inducible endothelial cell surface glycoprotein mediates melanoma adhesion. Science 1989; 246: 1303-1306. http://dx.doi.org/10.1126/ science. 2588007

[26] JOHNSON JP, STADE BG, HOLZMANN B, SCHW BLE W, RIETHM LLER G. De novo expression of intercellularadhesion molecule 1 in melanoma correlates with increased risk of metastasis. P Natl Acad Sci 1989; 86: 641-644. http:// dx.doi.org/10.1073/pnas.86.2.641

[27] BANKS R, GEARING A, HEMINGWAY I, NORFOLK D, PERREN $T$ et al. Circulating intercellular adhesion molecule-1 (ICAM-1), E-selectin and vascular cell adhesion molecule-1 (VCAM-1) in human malignancies. Br J Cancer 1993; 68: 122. http://dx.doi.org/10.1038/bjc.1993.298 
[28] KAGESHITA T, YOSHII A, KIMURA T, KURIYA N, ONO T et al. Clinical relevance of ICAM-1 expression in primary lesions and serum of patients with malignant melanoma. Cancer Res 1993; 53: 4927-4932. http://dx.doi.org/10.1016/09231811(93)90825-a

[29] MEYER P, STAPELMANN H, FRANK B, VARON R, BURWINKEL $B$ et al. Molecular genetic analysis of NBS1 in German melanoma patients. Melanoma Res 2007; 17: 109116. http://dx.doi.org/10.1097/CMR.0b013e3280dec638

[30] GRABSCH H, TAKENO S, PARSONS WJ, POMJANSKI N, BOECKING A et al. Overexpression of the mitotic checkpoint genes BUB1, BUBR1, and BUB3 in gastric cancer-association with tumour cell proliferation. J Pathol 2003; 200: 16-22. http://dx.doi.org/10.1002/path.1324

[31] RICKE RM, JEGANATHAN KB, VAN DEURSEN JM. Bub1 overexpression induces aneuploidy and tumor formation through Aurora B kinase hyperactivation. J Cell Biol 2011; 193: 1049-1064. http://dx.doi.org/10.1083/jcb.201012035

[32] RIKER AI, ENKEMANN SA, FODSTAD O, LIU S, REN S et al. The gene expression profiles of primary and metastatic melanoma yields a transition point of tumor progression and metastasis. BMC Med Genomics 2008; 1: 13. http://dx.doi. org/10.1186/1755-8794-1-13

[33] LAMERS F, VAN DER PLOEG I, SCHILD L, EBUS ME, KOSTER J et al. Knockdown of survivin (BIRC5) causes apoptosis in neuroblastoma via mitotic catastrophe. Endocr Relat Cancer 2011; 18: 657-668. http://dx.doi.org/10.1530/ERC-11-0207

[34] WINNEPENNINCKX V, LAZAR V, MICHIELS S, DESSEN P, STAS M et al. Gene expression profiling of primary cutaneous melanoma and clinical outcome. J Natl Cancer Inst 2006; 98: 472-482. http://dx.doi.org/10.1093/jnci/dji103

[35] KABBARAH O, NOGUEIRA C, FENG B, NAZARIAN RM, BOSENBERG $\mathrm{M}$ et al. Integrative genome comparison of primary and metastatic melanomas. PLoS One 2010; 5: e10770. http://dx.doi.org/10.1371/journal.pone.0010770
[36] KIM H-E, KIM D-G, LEE KJ, SON JG, SONG M-Y et al. Frequent amplification of CENPF, GMNN and CDK13 genes in hepatocellular carcinomas. PloS One 2012; 7: e43223. http:// dx.doi.org/10.1371/journal.pone.0043223

[37] GAO J, MA H, ZHOU Y, HU Z, ZHAI X et al. The association of polymorphisms of CDT1 and GMNN gene with the risk of breast cancer in Chinese women: a case-control analysis. Zhonghua Yi Xue Yi Chuan Xue Za Zhi 2006; 23: 544-547.

[38] COX DG, HANKINSON SE, HUNTER DJ. Polymorphisms of the AURKA (STK15/Aurora Kinase) gene and breast cancer risk (United States). Cancer Causes Control 2006; 17: 81-83. http://dx.doi.org/10.1007/s10552-005-0429-9

[39] HUYNH KM, KIM G, KIM D-J, YANG S-J, PARK S-M et al. Gene expression analysis of terminal differentiation of human melanoma cells highlights global reductions in cell cycle-associated genes. Gene 2009; 433: 32-39. http://dx.doi. org/10.1016/j.gene.2008.11.013

[40] MEDIC S, PEARCE RL, HEENAN PJ, ZIMAN M. Molecular markers of circulating melanoma cells. Pigment Cell Res 2007; 20: 80-91. http://dx.doi.org/10.1111/j.1600-0749 $.2006 .00356 . \mathrm{x}$

[41] KAUFFMANN A, ROSSELLI F, LAZAR V, WINNEPENNINCKX V, MANSUET-LUPO A et al. High expression of DNA repair pathways is associated with metastasis in melanoma patients. Oncogene 2008; 27: 565-573. http:// dx.doi.org/10.1038/sj.onc. 1210700

[42] KIECKER C, LUMSDEN A. Compartments and their boundaries in vertebrate brain development. Nat Rev Neurosci 2005; 6: 553-564. http://dx.doi.org/10.1038/nrn1702

[43] MATSUMOTO K, NISHIHARA S, KAMIMURA M, SHIRAISHI T, OTOGURO T et al. The prepattern transcription factor Irx2, a target of the FGF8/MAP kinase cascade, is involved in cerebellum formation. Nat neurosci 2004; 7: 605-612. http:// dx.doi.org/10.1038/nn1249 